\title{
The dynamics of the one-dimensional delta-function Bose gas
}

\author{
Craig A Tracy ${ }^{1}$ and Harold Widom ${ }^{2}$ \\ ${ }^{1}$ Department of Mathematics, University of California Davis, CA 95616, USA \\ ${ }^{2}$ Department of Mathematics, University of California, Santa Cruz, CA 95064, USA \\ E-mail: tracy@math.ucdavis.edu and widom@ucsc.edu
}

Received 18 August 2008

Published 21 October 2008

Online at stacks.iop.org/JPhysA/41/485204

\begin{abstract}
We give a method to solve the time-dependent Schrödinger equation for a system of one-dimensional bosons interacting via a repulsive delta function potential. The method uses the ideas of Bethe Ansatz but does not use the spectral theory of the associated Hamiltonian.
\end{abstract}

PACS number: 05.30.Jp

Mathematics Subject Classification: $82 \mathrm{C} 23$

\section{Introduction}

In this paper we give an alternative approach to solve the time-dependent Schrödinger equation for a system of one-dimensional bosons interacting via a delta function potential. This quantum many-body model, called the Lieb-Liniger model [6, 7], is 'exactly solvable' by means of Bethe Ansatz [2] in the sense that the ground-state energy [7], the excitation spectrum [6] as well as equilibrium thermodynamics [13] are known. (See [5, 10] for textbook treatments.) Current widespread interest in the Lieb-Liniger model has arisen because of its connection to ultracold gases confined in a quasi one-dimensional trap. Indeed, it has been recently shown that the Lieb-Liniger model for one-dimensional bosons 'can be rigorously derived via a scaling limit from a dilute three-dimensional Bose gas with arbitrary repulsive interaction potential of finite scattering length' [8]. These applications focus interest on the time-dependent solutions. For a review of these developments see [3].

Recall that the Lieb-Liniger $\delta$-function ( $N$-particle) Bose gas Hamiltonian is

$$
H=-\sum_{j=1}^{N} \frac{\partial^{2}}{\partial x_{j}^{2}}+2 c \sum_{j<k} \delta\left(x_{j}-x_{k}\right)
$$

where $c>0$ (the repulsive case) and $x_{j} \in \mathbb{R}^{3}$ Most work $[1,4,6,7,9,12]$ focuses on the eigenfunctions and eigenvalues of $H$ from which, in principle, any solution $\Psi(x ; t)$ of the

3 We choose units where $2 m=1$ and $\hbar=1$. 
time-dependent Schrödinger equation

$$
H \Psi=\mathrm{i} \frac{\partial \Psi}{\partial t},
$$

subject to the initial condition

$$
\Psi(x ; 0)=\Psi\left(x_{1}, \ldots, x_{N} ; 0\right)=\psi_{0}\left(x_{1}, \ldots, x_{N}\right),
$$

may be constructed ${ }^{4}$. In practice, due to the complexity of the Bethe eigenfunctions and the nonlinear Bethe equations, it is difficult to analyze time-dependent solutions whose initial conditions are not eigenfunctions. We give here an alternative to the spectral method for solving (2).

Since different physical conditions require different choices of the initial wavefunction $\psi_{0}$, we develop here a flexible mathematical structure to incorporate these different choices. Suppose we solve (2) with the initial condition

$$
\psi_{\delta}(x)=\psi_{\delta}\left(x_{1}, \ldots, x_{N}\right)=\frac{1}{N !} \sum_{\sigma \in \mathbb{S}_{N}} \prod_{j=1}^{N} \delta\left(x_{\sigma(j)}-y_{j}\right)
$$

where $\mathbb{S}_{N}$ is the permutation group acting on $\{1, \ldots, N\}$. Here $y_{j} \in \mathbb{R}$ are fixed and without loss of generality we may assume

$$
y_{1}<y_{2}<\cdots<y_{N} .
$$

If $\Psi_{\delta}(x, y ; t)$ denotes this solution, then the solution to (2) satisfying (3) is

$$
\Psi(x ; t)=\int_{\mathbb{R}^{N}} \Psi_{\delta}(x, y ; t) \psi_{0}(y) \mathrm{d} y
$$

( $\left.\mathrm{d} y=\mathrm{d} y_{1} \cdots \mathrm{d} y_{N}\right)$. The subject of this paper is $\Psi_{\delta}$, a Green's function for (2).

We remark that there are two natural choices for the initial wavefunction $\psi_{0}$. The free particle Gaussian wavefunction localized at $y$ is ${ }^{5}$

$$
\varphi_{a}(x ; y, p)=\frac{1}{(2 \pi)^{1 / 4} \sqrt{a}} \mathrm{e}^{-(x-y)^{2} /\left(4 a^{2}\right)} \mathrm{e}^{\mathrm{i} p x} .
$$

Thus an initial condition where the particles are separated and free is

$$
\psi_{\text {free }}(x)=c_{N} \sum_{\sigma \in \mathbb{S}_{N}} \prod_{j=1}^{N} \varphi_{a}\left(x_{\sigma(j)} ; y_{j}, p_{j}\right)
$$

where $c_{N}$ is a normalization constant.

A second choice is for the Bose gas to be initially confined to a subset of $\mathbb{R}$ and in its ground state. Solving (2) with this initial condition corresponds to the confined Bose gas freely expanding into all of $\mathbb{R}$. The ground-state wavefunction for confinement to $\mathbb{R}^{+}$has been computed by Gaudin [4] and confinement to a hard wall box by Batchelor et al [1].

We now recall a well-known [7, 10] reformulation of the problem. Since we seek symmetric solutions, it is sufficient to solve (2) in the region

$$
\mathcal{R}:-\infty<x_{1} \leqslant x_{2} \leqslant \cdots \leqslant x_{N}<\infty .
$$

In the interior of $\mathcal{R}$, e.g. $\mathcal{R}^{o}: x_{1}<x_{2}<\cdots<x_{N}$, the $\delta$-functions are zero and we have the free Schrödinger equation

$$
-\sum_{i} \frac{\partial^{2} \Psi_{\delta}}{\partial x_{i}^{2}}=\mathrm{i} \frac{\partial \Psi_{\delta}}{\partial t}
$$

\footnotetext{
$4 \psi_{0}$ is a given symmetric function of the coordinates $x_{j}$.

5 Thus $\left|\varphi_{a}(x ; y, p)\right|^{2}$ is the Gaussian distribution centered at $y$ with variance $a^{2}$. Solving the time-dependent free Schrödinger equation shows that $\langle x\rangle=2 p t$; and hence, $2 p$ is the classical velocity. (Recall $2 m=1$.)
} 
That is the effect of the $\delta$-functions are confined to the boundary of $\mathcal{R}$, e.g. on the hyperplanes $x_{j}=x_{j+1}$, and their effect can be formulated as a boundary condition on the hyperplanes (we also use the fact that $\Psi_{\delta}$ is a symmetric function):

$$
\left.\left(\frac{\partial}{\partial x_{j+1}}-\frac{\partial}{\partial x_{j}}\right) \Psi_{\delta}\right|_{x_{j+1}=x_{j}}=\left.c \Psi_{\delta}\right|_{x_{j+1}=x_{j}} .
$$

Thus the problem is to solve (7) in $\mathcal{R}^{o}$ subject to the boundary conditions (8) and the initial condition in $\mathcal{R}$

$$
\Psi_{\delta}(x ; 0)=\prod_{j=1}^{N} \delta\left(x_{j}-y_{j}\right)
$$

where $y_{j}$ satisfy (5) and we have dropped the normalization constant $N$ ! since it can be incorporated at the end. The solution $\Psi_{\delta}$ is given below in (14).

\section{Bethe Ansatz}

We now explain how the ideas of Bethe Ansatz [2, 7] can be employed to solve the timedependent problem without using the spectral theory of the operator $H$. Since we avoid the eigenvalue problem, there are no Bethe equations in this approach ${ }^{6}$. To set the notation and to see the argument in its simplest form, we first solve $N=2$.

\section{1. $N=2$}

It is elementary to verify that

$$
\int_{\mathbb{R}} \int_{\mathbb{R}} A\left(k_{1}, k_{2}\right) \mathrm{e}^{\mathrm{i}\left(k_{1} x_{1}+k_{2} x_{2}\right)} \mathrm{e}^{-\mathrm{i}\left(\varepsilon\left(k_{1}\right)+\varepsilon\left(k_{2}\right)\right) t} \mathrm{~d} k_{1} \mathrm{~d} k_{2}
$$

with

$$
\varepsilon(k)=k^{2}
$$

solves (7) in $x_{1}<x_{2}$. The insight from Bethe Ansatz is to add to this solution another solution with the integration variables permuted,

$$
\int_{\mathbb{R}} \int_{\mathbb{R}}\left[A_{12}\left(k_{1}, k_{2}\right) \mathrm{e}^{\mathrm{i}\left(k_{1} x_{1}+k_{2} x_{2}\right)}+A_{21}\left(k_{1}, k_{2}\right) \mathrm{e}^{\mathrm{i}\left(k_{2} x_{1}+k_{1} x_{2}\right)}\right] \mathrm{e}^{-\mathrm{i}\left(\varepsilon\left(k_{1}\right)+\varepsilon\left(k_{2}\right)\right) t} \mathrm{~d} k_{1} \mathrm{~d} k_{2},
$$

so that the boundary condition (8) can be applied pointwise to the above integrand. The result is the condition

$$
\mathrm{i} k_{2} A_{12}+\mathrm{i} k_{1} A_{21}-\mathrm{i} k_{1} A_{12}-\mathrm{i} k_{2} A_{21}=c\left(A_{12}+A_{21}\right),
$$

or equivalently,

$$
A_{21}=-\frac{c-\mathrm{i}\left(k_{2}-k_{1}\right)}{c+\mathrm{i}\left(k_{2}-k_{1}\right)} A_{12} .
$$

We set

$$
S_{\alpha \beta}=S_{\alpha \beta}\left(k_{\alpha}-k_{\beta}\right)=-\frac{c-\mathrm{i}\left(k_{\alpha}-k_{\beta}\right)}{c+\mathrm{i}\left(k_{\alpha}-k_{\beta}\right)}
$$

so that the above reads $A_{21}=S_{21} A_{12}$. It is also convenient to set

$$
S(k)=-\frac{c-\mathrm{i} k}{c+\mathrm{i} k} \text {. }
$$

6 The methods here are an adaptation of the methods of [11]. 
Observe that $S(k)$ extends to a holomorphic function in the lower half-plane and so its Fourier transform, $\hat{S}(z)$, is supported in $[0, \infty)$.

With the initial condition (9) in mind, we choose

$$
A_{12}\left(k_{1}, k_{2}\right)=\mathrm{e}^{-\mathrm{i}\left(k_{1} y_{1}+k_{2} y_{2}\right)}
$$

so the above solution becomes

$\int_{\mathbb{R}} \int_{\mathbb{R}}\left[\mathrm{e}^{\mathrm{i}\left(k_{1}\left(x_{1}-y_{1}\right)+k_{2}\left(x_{2}-y_{2}\right)\right)}+S_{21}\left(k_{2}, k_{1}\right) \mathrm{e}^{\mathrm{i}\left(k_{2}\left(x_{1}-y_{2}\right)+k_{1}\left(x_{2}-y_{1}\right)\right)}\right] \mathrm{e}^{-\mathrm{i}\left(\varepsilon_{k_{1}}+\varepsilon_{k_{2}}\right) t} \mathrm{~d} k_{1} \mathrm{~d} k_{2}$

where from now on $d k_{j} \rightarrow d k_{j} / 2 \pi$.

At $t=0$ the first term evaluates to $\delta\left(x_{1}-y_{1}\right) \delta\left(x_{2}-y_{2}\right)$. Thus we must show

$$
\int_{\mathbb{R}} \int_{\mathbb{R}} S\left(k_{2}-k_{1}\right) \mathrm{e}^{\mathrm{i} k_{1}\left(x_{2}-y_{1}\right)+i k_{2}\left(x_{1}-y_{2}\right)} \mathrm{d} k_{1} \mathrm{~d} k_{2}=0
$$

for $y_{1}<y_{2}$ and $x_{1} \leqslant x_{2}$ for the solution (12) to satisfy the initial condition (9). Making the change of variables $k_{2} \rightarrow k_{2}+k_{1}$ and $k_{1} \rightarrow k_{1}$ in the above integral, we have (after performing the resulting integration over $k_{1}$ )

$\delta\left(x_{1}+x_{2}-y_{1}-y_{2}\right) \int_{\mathbb{R}} S\left(k_{2}\right) \mathrm{e}^{\mathrm{i} k_{2}\left(x_{1}-y_{2}\right)} \mathrm{d} k_{2}=\delta\left(x_{1}+x_{2}-y_{1}-y_{2}\right) \hat{S}\left(x_{1}-y_{2}\right)$.

Now $\hat{S}\left(x_{1}-y_{2}\right)$ is nonzero only in the region

$$
x_{1}-y_{2} \geqslant 0
$$

and the delta function requires that $x_{1}+x_{2}=y_{1}+y_{2}$ for a nonzero contribution. But in $\mathcal{R}$

$$
2 x_{1} \leqslant x_{1}+x_{2}=y_{1}+y_{2}<2 y_{2},
$$

i.e. $x_{1}<y_{2}$. Hence the integral in the region $\mathcal{R}$ is zero, and thus, we conclude (12) is the sought after solution $\Psi_{\delta}(x)$ for $N=2$.

\subsection{General $N$}

Let $\sigma \in \mathcal{S}_{N}$ be a permutation of $\{1, \ldots, N\}$. Recall that an inversion in a permutation $\sigma$ is an ordered pair $\{\sigma(i), \sigma(j)\}$ in which $i<j$ and $\sigma(i)>\sigma(j)$. We set

$$
A_{\sigma}=\prod\left\{S_{\alpha \beta}:\{\alpha, \beta\} \text { is an inversion in } \sigma\right\}
$$

where $S_{\alpha \beta}$ is defined by (10). Thus, for example, $A_{231}=S_{21} S_{31}$ and $A_{i d}=1$. We claim the solution that satisfies (7) with boundary conditions (8) and the initial condition (9) is

$$
\Psi_{\delta}(x ; t)=\sum_{\sigma \in \mathcal{S}_{N}} \int_{\mathbb{R}} \cdots \int_{\mathbb{R}} A_{\sigma} \prod_{j=1}^{N} \mathrm{e}^{\mathrm{i} k_{\sigma(j)}\left(x_{j}-y_{\sigma(j)}\right)} \mathrm{e}^{-\mathrm{i} t \sum_{j} \varepsilon\left(k_{j}\right)} \mathrm{d} k_{1} \cdots \mathrm{d} k_{N} .
$$

First, it is clear that (14) satisfies (7) in $\mathcal{R}^{o}$. As demonstrated in [7], if $T_{i} \sigma$ denotes $\sigma$ with the entries $\sigma(i)$ and $\sigma(i+1)$ interchanged, the boundary conditions will be satisfied provided that

$$
A_{T_{i} \sigma}=S_{\sigma(i+1) \sigma(i)} A_{\sigma}
$$

for all $\sigma$. Let us see why this relation holds. Let $\alpha=\sigma(i), \beta=\sigma(i+1)$, and suppose $\alpha>\beta$. Then $\{\alpha, \beta\}$ is an inversion for $\sigma$ but not for $T_{i} \sigma$, so $S_{\alpha \beta}$ is a factor in $A_{\sigma}$ but not in $A_{T_{i} \sigma}$, and all other factors are the same. Therefore, using $S_{\alpha \beta} S_{\beta \alpha}=1$, we have

$$
A_{T_{i} \sigma}=S_{\beta \alpha} A_{\sigma}=S_{\sigma(i+1) \sigma(i)} A_{\sigma} .
$$

The same identity holds immediately if $\beta>\alpha$, since $\{\beta, \alpha\}$ is an inversion for $T_{i} \sigma$ but not for $\sigma$. 
As in the $N=2$ case, the term corresponding to the identity permutation in (14) satisfies the initial condition (9). Thus to complete the proof we must show

$$
\sum_{\sigma \in \mathcal{S}_{N}, \sigma \neq i d} \int_{\mathbb{R}} \cdots \int_{\mathbb{R}} A_{\sigma} \prod_{j=1}^{N} \mathrm{e}^{\mathrm{i} k_{\sigma(j)}\left(x_{j}-y_{\sigma(j)}\right)} \mathrm{d} k_{1} \cdots \mathrm{d} k_{N}=0 .
$$

Let $I(\sigma)$ denote the integral corresponding to $\sigma$ term in the above sum. Recalling the definition (11) of $S$, the integrand for $I(\sigma)$ becomes

$$
\prod_{j=1}^{N} \mathrm{e}^{\mathrm{i} k_{\sigma(j)}\left(x_{j}-y_{\sigma(j)}\right)} \prod\left\{S\left(k_{\alpha}-k_{\beta}\right):\{\alpha, \beta\} \text { is an inversion in } \sigma\right\} .
$$

We again use the fact that $S(k)$ extends analytically into the lower half-plane.

If a number $\gamma$ appears on the left side of an inversion and never on the right side then in the integrand it appears only in factors of the form $S\left(k_{\gamma}-k_{\beta}\right)$ and so integrating with respect to $k_{\gamma}$ shows that the integrand is zero unless

$$
x_{\sigma^{-1}(\gamma)} \geqslant y_{\gamma} .
$$

Similarly, if a number $\delta$ appears on the right side of an inversion and never on the left side then in the integrand it appears only in factors of the form $S\left(k_{\alpha}-k_{\delta}\right)$ and so integrating with respect to $k_{\delta}$ shows that the resulting integrand is zero unless

$$
x_{\sigma^{-1}(\delta)} \leqslant y_{\delta} .
$$

We show $I(\sigma)=0$ by induction on $N$. If $\sigma(1)=1$ then 1 does not appear in an inversion, we can integrate with respect to $k_{1}$, and we are reduced to the case $N-1$. So assume $\sigma(1)=\gamma>1$. Then $(\gamma, 1)$ is an inversion and there is no inversion of the form $(\alpha, \gamma)$ because $\gamma$ appears in slot 1 . So we can apply (15) with this $\gamma$ and get the resulting integrand is zero unless

$$
x_{1} \geqslant y_{\gamma} .
$$

Next we observe as before that $(\gamma, 1)$ is an inversion, but now that 1 , which appears on the right side of the inversion, cannot appear on the left side of an inversion (obviously). So we can apply (16) with $\delta=1$ and get

$$
x_{\sigma^{-1}(1)} \leqslant y_{1} \text {. }
$$

Since $y_{1}<y_{\gamma}$ the two inequalities give $x_{\sigma^{-1}(1)}<x_{1}$, which cannot happen. This completes the proof of (14).

The limit of impenetrable bosons is the limit $c \rightarrow \infty$. In this limit $S_{\alpha \beta} \rightarrow-1$ and $A_{\sigma} \rightarrow(-1)^{i n v(\sigma)}$ where $\operatorname{inv}(\sigma)$ is the number of inversions in $\sigma$. Thus in $\mathcal{R}$

$$
\Psi_{\delta}(x ; t) \rightarrow \Psi_{\infty}(x ; t)=\int_{\mathbb{R}} \cdots \int_{\mathbb{R}} \operatorname{det}\left(\mathrm{e}^{\mathrm{i} k_{\alpha}\left(x_{\beta}-y_{\alpha}\right)-i t \varepsilon_{k_{\alpha}}}\right) \mathrm{d} k_{1} \cdots \mathrm{d} k_{N}
$$

and the extension to $\mathbb{R}^{N}$ follows by requiring $\Psi_{\infty}$ to be a symmetric function of $x_{j}$.

\section{Acknowledgments}

This work was supported by the National Science Foundation under grants DMS-0553379 (first author) and DMS-0552388 (second author). 


\section{References}

[1] Batchelor M T, Guan X W, Oelkers N and Lee C 2005 The 1 D interacting Bose gas in a hard wall box J. Phys. A.: Math. Gen. 38 7787-806

[2] Bethe H A 1931 On the theory of metals, I. Eigenvalues and eigenfunctions of a linear chain of atoms (German) Zeits. Phys. 1931 205-26

Bethe H A 1996 Selected Works of Hans A. Bethe With Commentary (Singapore: World Scientific (English translation appears)) pp 689-716

[3] Bloch I, Dalibard J and Zwerger W 2008 Many-body physics with ultracold gases Rev. Mod. Phys. 80 885-964

[4] Gaudin M 1971 Boundary energy of a Bose gas in one dimension Phys. Rev. A 4 386-94

[5] Korepin V E, Bogoliubov N M and Izergin A G 1993 Quantum Inverse Scattering Method and Correlation Functions (Cambridge: Cambridge University Press)

[6] Lieb E H 1963 Exact analysis of an interacting Bose gas. II: the excitation spectrum Phys. Rev. 130 1616-24

[7] Lieb E H and Liniger W 1963 Exact analysis of an interacting Bose gas. I: the general solution and the ground state Phys. Rev. 130 1605-16

[8] Seiringer R and Yin J The Lieb-Liniger model as a limit of dilute bosons in three dimensions Commun. Math. Phys. doi: 10.1007/s00220-008-0521-6

[9] Sutherland B 1968 Further results for the many-body problem in one dimension Phys. Rev. Lett. 20 98-100

[10] Sutherland B 2004 Beautiful Models: 70 Years of Exactly Solved Quantum Many-Body Problems (Singapore: World Scientific)

[11] Tracy C A and Widom H 2008 Integral formulas for the asymmetric simple exclusion process Commun. Math. Phys. 132 815-44

[12] Yang C N 1967 Some exact results for the many-body problem in one dimension with replusive delta-function interaction Phys. Rev. Lett. 19 1312-5

[13] Yang C N and Yang C P 1969 Thermodynamics of a one-dimensional system of bosons with replusive deltafunction interaction J. Math. Phys. 10 1115-22 